\title{
Precursor Wave Amplification by Ion-Electron Coupling through Wakefield in Relativistic Shocks
}

\author{
Masanori Iwamoto ${ }^{1}\left(\mathbb{1}\right.$, Takanobu Amano ${ }^{1}$, Masahiro Hoshino ${ }^{1}$ (i), Yosuke Matsumoto ${ }^{2}$ Jacek Niemiec $^{3}$ (i), Arianna Ligorini $^{3}$, \\ Oleh $\operatorname{Kobzar}^{3}$ (D), and Martin Pohl ${ }^{4,5}$ (DD \\ ${ }^{1}$ Department of Earth and Planetary Science, University of Tokyo, 7-3-1 Hongo, Bunkyo-ku, Tokyo 113-0033, Japan; iwamoto@eps.s.u-tokyo.ac.jp \\ ${ }^{2}$ Department of Physics, Chiba University, 1-33 Yayoi, Inage-ku, Chiba, Chiba 263-8522, Japan \\ ${ }^{3}$ Instytut Fizyki Jadrowej PAN, ul. Radzikowskiego 152, 31-342 Kraków, Poland \\ ${ }^{4}$ Institut für Physik und Astronomie, Universität Potsdam Karl-Liebknecht-Strasse 24/25, D-14476 Potsdam, Germany \\ ${ }^{5}$ DESY, Platanenallee 6, D-15738 Zeuthen, Germany \\ Received 2019 August 21; revised 2019 September 5; accepted 2019 September 7; published 2019 September 25
}

\begin{abstract}
We investigated electromagnetic precursor wave emission in relativistic shocks by using two-dimensional particlein-cell simulations. We found that the wave amplitude is significantly enhanced by a positive feedback process associated with ion-electron coupling through the wakefields for high magnetization. The wakefields collapse during the nonlinear process of the parametric decay instability in the near-upstream region, where nonthermal electrons and ions are generated. The intense coherent emission and the particle acceleration may operate in highenergy astrophysical objects.
\end{abstract}

Unified Astronomy Thesaurus concepts: Shocks (2086); Plasma physics (2089); Cosmic rays (329); High energy astrophysics (739)

\section{Introduction}

The acceleration mechanism for generating ultra-highenergy cosmic rays (UHECRs) with energies above $10^{18} \mathrm{eV}$ is one of the most important unsolved problems in astrophysics. Relativistic shocks in extragalactic astrophysical objects such as jets from active galactic nuclei and gamma-ray bursts are considered to be efficient acceleration sites (e.g., Piran 2005; Marscher 2006) and are thus likely sources for UHECRs (e.g., Hillas 1984; Biermann \& Strittmatter 1987; Milgrom \& Usov 1995; Vietri 1995; Waxman 1995). Observations of anisotropy in the arrival direction of the UHECRs favor the extragalactic origin (e.g., Abbasi et al. 2014; Aab et al. $2015,2017,2018)$. In addition, the recent observation by IceCube reported that a high-energy neutrino event associated with the UHECRs correlates in direction and time with a gamma-ray flare from the blazar (Aartsen et al. 2018).

In relativistic perpendicular shocks, large-amplitude electromagnetic waves are excited by synchrotron maser instability (SMI). Since the precursor waves are intense and coherent, fast radio bursts (FRBs) may be attributed to the SMI in relativistic shocks (e.g., Lyubarsky 2014; Metzger et al. 2019; Plotnikov \& Sironi 2019). The SMI induces extraordinary mode (Xmode) waves and thus the polarization of the precursor waves also supports the FRB model (see Plotnikov \& Sironi 2019). The wave emission has been widely studied by means of onedimensional (1D) particle-in-cell (PIC) simulations (e.g., Langdon et al. 1988; Hoshino \& Arons 1991; Gallant et al. 1992; Hoshino et al. 1992; Amato \& Arons 2006). Lyubarsky (2006) showed that electrons lag behind ions inside such largeamplitude waves and that a longitudinal electric field is excited in the wake of the precursor wave. Hoshino (2008) extended the work and demonstrated that the pump electromagnetic wave decays into a Langmuir wave via parametric decay instability (PDI; e.g., Mima \& Nishikawa 1984; Kruer 1988). PDI is a wave-wave interaction and large-amplitude electromagnetic waves are subject to it because they exert radiation pressure on plasmas and induce forward-propagating compressive fluctuations. When Raman scattering, which is an inelastic scattering process of light, works in plasmas, the PDI produces Langmuir waves. He found that nonthermal particles are generated in the manner analogous to wakefield acceleration (WFA) during the nonlinear process of the Langmuir wave collapse. The WFA is first proposed in laboratory plasmas (Tajima \& Dawson 1979) and later applied to UHECR acceleration (e.g., Chen et al. 2002; Arons 2003; Murase et al. 2009). In laboratory plasmas, wakefield, which is a longitudinal electrostatic wave, is excited by an ultra-intense laser pulse. Some electrons are preferentially accelerated by the wakefield via Landau resonance. Previous laser-plasma experiments (Kuramitsu et al. 2011a, 2011b) and simulations (Kuramitsu et al. 2008, 2012; Liu et al. 2017, 2018, 2019) showed that the WFA produces a power-law distribution with a spectral index of 2. Therefore, the WFA in the context of relativistic shocks is a promising candidate for UHECR acceleration.

Recently, our high-resolution two-dimensional (2D) PIC simulations in pair plasmas (Iwamoto et al. 2017, 2018) showed that the wave emission continues even well after Weibel instability (Fried 1959; Weibel 1959) becomes active at the shock front. The precursor waves are large enough to disturb the upstream plasma and induces transverse density filaments extended well ahead of the shock front (see also Plotnikov et al. 2018). The interaction between the strong precursor waves and the upstream plasma is not negligible even in pair plasmas (see also Lyubarsky 2018). Although our results for pair plasmas are favorable for the WFA scenario in relativistic shocks, we could not directly demonstrate it because finite mass difference of two opposite charges is essential for exciting the wakefield.

In this Letter, by performing a 2D PIC simulation of ionelectron shocks, we demonstrate that the wakefield is indeed induced by the large-amplitude precursor waves. Especially for high magnetization, the wave amplitude is significantly amplified due to a positive feedback process associated with 
ion-electron coupling. Nonthermal electrons and ions are generated during the nonlinear collapse of the wakefield and the particle energy spectrum shows a power-law distribution. Our self-consistent simulations of multidimensional relativistic shocks provide insights into the physics of wave-plasma interaction and particle acceleration in high-energy astrophysical objects.

\section{Simulation Setup}

We employed a fully relativistic electromagnetic PIC code (Matsumoto et al. 2013, 2015), which enables us to follow long-term evolution by minimizing the effect of numerical Cherenkov instability (Ikeya \& Matsumoto 2015). The basic configuration is nearly identical to our previous simulations (Iwamoto et al. 2017). The essential difference is a finite ion-toelectron mass ratio $m_{i} / m_{e}=50$. The simulation domain is in the $x-y$ plane and the periodic boundary condition is applied in the $y$ direction. We consider purely perpendicular shocks and the ambient magnetic field $B_{1}$ is in the $z$ direction. The cold plasma flow (with zero thermal spread of electrons and ions) is injected from the right-hand boundary with the bulk Lorentz factor $\gamma_{1}=40$ toward the left-hand reflecting wall at $x=0$. The interaction between incoming and reflected particles triggers the shock propagating toward the $+x$ direction. The upstream particle number per cell $N_{1}$ is set as $N_{1} \Delta x^{2}=64$ for each particle species. The gird size $\Delta x$ and the time steps $\Delta t$ are fixed to $\Delta x /\left(c / \omega_{p e}\right)=1 / 40$ and $\omega_{p e} \Delta t=1 / 40$, where $\omega_{p e}=\sqrt{4 \pi N_{1} e^{2} / \gamma_{1} m_{e}}$ is the proper electron plasma frequency. Note that our simulations with the CFL number $c \Delta t /$ $\Delta x=1$ are numerically stable because of an implicit Maxwell solver used in our code. The basic structure of relativistic magnetized shocks is characterized by the ratio of the Poynting flux to the upstream bulk kinetic energy flux $\sigma_{s}=B_{1}^{2} / 4 \pi \gamma_{1} N_{1} m_{s} c^{2}$, where the subscript $s=i, e$ represents particle species. Our shock simulations are performed for values of $\sigma_{e}=5,1$, and 0.1 . Note that $\sigma_{i}=\left(m_{e} / m_{i}\right) \sigma_{e}$ is always satisfied and $\sigma_{i}$ is then $0.1,2 \times 10^{-2}$, and $2 \times 10^{-3}$, respectively. The grid size in the unit of the electron gyroradius is given by $\Delta x /\left(c / \omega_{c e}\right)=\sqrt{\sigma_{e}} / 40$, where $\omega_{c e}=e B_{1} / \gamma_{1} m_{e} c=\sqrt{\sigma_{e}} \omega_{p e}$ is the electron cyclotron frequency, and thus it varies from $\sim 0.06$ at $\sigma_{e}=5$ to $\sim 0.008$ at $\sigma_{e}=0.1$. We used the different simulation box sizes for each magnetization and the number of grids is $N_{x} \times N_{y}=80,000 \times 1600, \quad 80,000 \times 800, \quad$ and $200,000 \times 1600$, respectively. The ambient magnetic field is fixed in the $z$ direction (i.e., out-of-plane configuration) throughout this study. Iwamoto et al. (2018) show that in the in-plane configuration, ordinary mode (O-mode) waves as well as $\mathrm{X}$-mode waves are excited due to fluctuations along the ambient magnetic field. For $\sigma_{e} \gtrsim 10^{-1}$, the X-mode waves are dominant over the O-mode waves and the $\mathrm{X}$-mode wave amplitude is almost equal to that in the out-of-plane configuration. Therefore, we focus on the out-of-plane configuration in this study.

\section{Shock Structure}

Figure 1 shows the global structure of the shock at $\omega_{p e} t=2000$ in the case of relatively high magnetization: $\sigma_{e}=5$. From top to bottom, the out-of-plane magnetic field $B_{z}$, the $1 \mathrm{D}$ cut of $B_{z}$ at $y /\left(c / \omega_{p e}\right)=20$, the longitudinal electric field $E_{x}$, the $y$-averaged electric field $\left\langle E_{x}\right\rangle$, and the electron and ion phase space densities $x-u_{x s}$ integrated over the $y$ direction are shown. Here $u_{1}=\sqrt{\gamma_{1}^{2}-1} \simeq 40$ is the bulk four velocity of injected particles. The large-amplitude electromagnetic waves are clearly seen in the upstream. The precursor wave induces a large-scale longitudinal electric field, which is socalled wakefield as already discussed by Hoshino (2008). The linear theory of the PDI via forward Raman scattering process shows that the wavelength of the Langmuir wave measured in the simulation frame can be estimated by $\lambda_{L} /\left(c / \omega_{p e}\right) \simeq 4 \pi \gamma_{1} \simeq 500$ (see, e.g., Mima \& Nishikawa 1984; Kruer 1988; Hoshino 2008). This estimate comes from the frequency and wavevector matching conditions of the three waves under the assumption that the pump wave frequency is sufficiently large. Considering the sinusoidal part of the wakefield in the region $1350 \lesssim x /\left(c / \omega_{p e}\right) \lesssim 1850$, this estimate gives a good agreement with our simulation result. The wakefield breaks up in the region $1150 \lesssim x /\left(c / \omega_{p e}\right) \lesssim$ 1350 , indicating that the PDI enters the nonlinear phase. The precursor waves also become turbulent there and the transverse filamentary structure is generated. Both electrons and ions are strongly heated and accelerated in the turbulent region.

\section{Precursor Wave Amplification}

The modulation of the precursor wave amplitude is clearly visible in Figure 1, indicating that the amplitude changes in time. Figure 2 shows temporal evolution of the precursor wave energy normalized by the upstream electron kinetic energy $\epsilon_{p}=\delta B^{2} / 4 \pi \gamma_{1} N_{1} m_{e} c^{2}$, the wakefield $\left\langle E_{x}\right\rangle$, and the electron bulk velocity $\left\langle u_{x e}\right\rangle$. Here $\delta B=B_{z}-B_{1}$ is the wave component of the magnetic field. Note that positive longitudinal electric fields are excited in an initial phase of the PDI because electrons lag behind ions inside intense precursor waves (see Lyubarsky 2006). One can find that $\epsilon_{p}$ increases in time and that the wave amplification are coincident with an increase of the magnitude of $\left\langle E_{x}\right\rangle$ and $\left\langle u_{x e}\right\rangle$. This observation indicates that the accelerated electrons cause the increase of the wave amplitude, which initiates further acceleration of electrons through the wakefield, and that the positive feedback process discussed in the earlier studies (Lyubarsky 2006; Hoshino 2008) operates in 2D as well. Note that the wave emission efficiency is mainly controlled by electrons because ions do not contribute to the electromagnetic wave emission via the SMI (Hoshino \& Arons 1991). Initially, the precursor waves are emitted by consuming the free energy of electrons with an initial Lorentz factor of $\gamma_{1}$. The precursor waves then induce a wakefield via the PDI in the immediate upstream of the shock. The incoming electrons are gradually accelerated or decelerated by the wakefield on their way to the shock. When the accelerated electrons hit the shock, the precursor wave may be amplified because of an increased free energy available for the SMI. The amplified precursor waves induce a stronger wakefield which in turn accelerates the upstream incoming electrons even further. If this self-reinforcing cycle operates sufficiently rapidly, the precursor wave amplitude continuously grows over time. The ion kinetic energy is converted into the electron through the wakefield during this positive feedback process and the SMI indirectly consumes the ion kinetic energy. The precursor wave amplitude takes the maximum value when the electron kinetic energy achieves equipartition with the ion (Lyubarsky 2006).

Left panel of Figure 3 shows $\sigma_{e}$ dependence of the normalized wave energy $\epsilon_{p}$ in red. We measured the average 


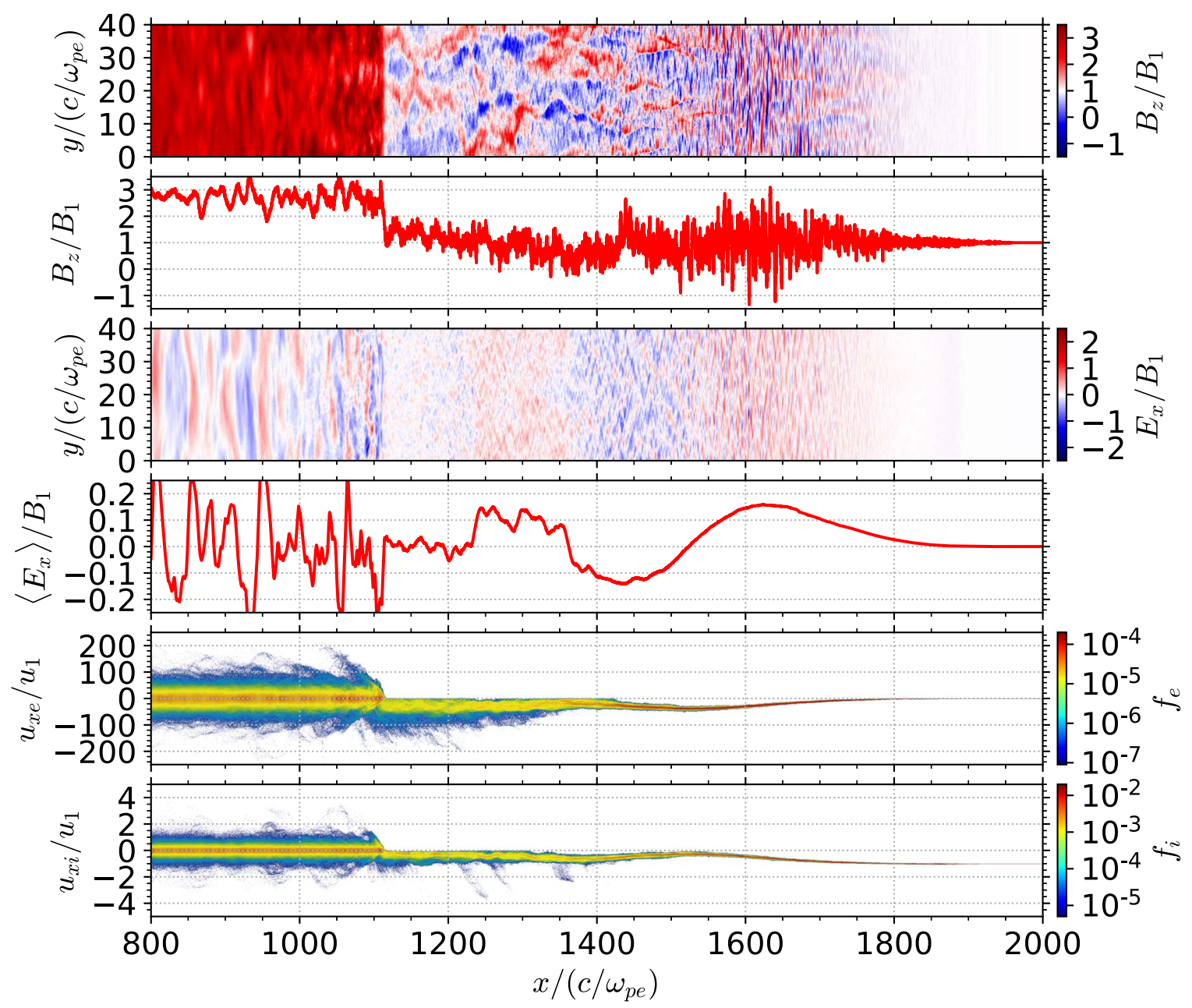

Figure 1. Shock structure for $\sigma_{e}=5$ at $\omega_{p e} t=2000$. The out-of-plane magnetic field $B_{z}$, the 1D profile of $B_{z}$, the longitudinal electric field $E_{x}$, the $y$-averaged electric field $\left\langle E_{x}\right\rangle$, and the phase space plots in the $x-u_{x s}$ plane for electrons and ions are shown.

wave energy inside the laminar wakefield (e.g., $1350 \leqslant x /\left(c / \omega_{p e}\right) \leqslant 1850$ for $\left.\sigma_{e}=5\right)$ in order to evaluate the effect of the positive feedback process on the wave emission efficiency and exclude the nonlinear effect of the PDI. The dots and solid lines indicate ion-electron and pair shock simulation results, respectively. We performed 1D ion-electron shock simulations for comparison and the results are shown in blue. The wave energy in $2 \mathrm{D}$ is systematically smaller than that in 1D because of the inhomogeneity along the shock surface (Iwamoto et al. 2017, 2018).

The wave energy in ion-electron plasmas exceeds that in pair plasmas for both 1D and 2D except for the 2D run with $\sigma_{e}=0.1$. We attribute this amplification over the pair plasmas to the positive feedback process, which does not work in pair plasmas due to the absence of the wakefield. If the electron kinetic energy achieves equipartition with the ion during the positive feedback process, the incoming electron bulk Lorentz factor becomes larger by a factor of $m_{i} / m_{e}$ than the initial. In other words, the effective $\sigma_{e}$ decreases down to $\left(m_{e} / m_{i}\right) \sigma_{e}$, whereas $\epsilon_{p}$ increases up to $\left(m_{i} / m_{e}\right) \epsilon_{p}$ during the amplification phase. To confirm the hypothesis that the observed amplified emission is due to the positive feedback, we have also shown the emission efficiency measured in pair plasmas rescaled by appropriate factors:

$$
\epsilon_{p}^{\prime}\left(\sigma_{e}\right)=\frac{1}{2} \frac{m_{i}}{m_{e}} \epsilon_{p}\left(\frac{m_{i}}{m_{e}} \sigma_{e}\right)
$$

in the left panel of Figure 3 with the dashed lines. Here the factor of $1 / 2$ is introduced because the wave amplitude in ionelectron plasmas was obtained by averaging over the laminarwakefield region. The wave emission efficiency measured in pair plasmas $\epsilon_{p}$ can be converted to that in ion-electron plasmas $\epsilon_{p}^{\prime}$ according to Equation (1). The $\sigma_{e}$ dependence of the wave energy in ion-electron plasmas is in rough agreement with the positive feedback model associated with the ion-electron coupling except for the 2D run with $\sigma_{e}=0.1$. Although the 2D run with $\sigma_{e}=1$ seems to be deviated from the rescaled emission efficiency, the difference is at most a factor of three. Furthermore, we investigated the mass ratio dependence of the 1D wave energy. We fixed the upstream $\sigma_{i}=\left(m_{e} / m_{i}\right) \sigma_{e}$ and measured the wave energy $\epsilon_{p}$ in the same manner. Our simulations in pair plasmas show that $\epsilon_{p}$, which is the energy conversion rate from the free energy into the precursor wave energy, is constant for a given $\sigma_{e}$. Since the free energy available for the SMI increases with the mass ratio in ionelectron plasmas due to the positive feedback process, the wave energy $\epsilon_{p}$ should be a linear function of the mass ratio. As can 


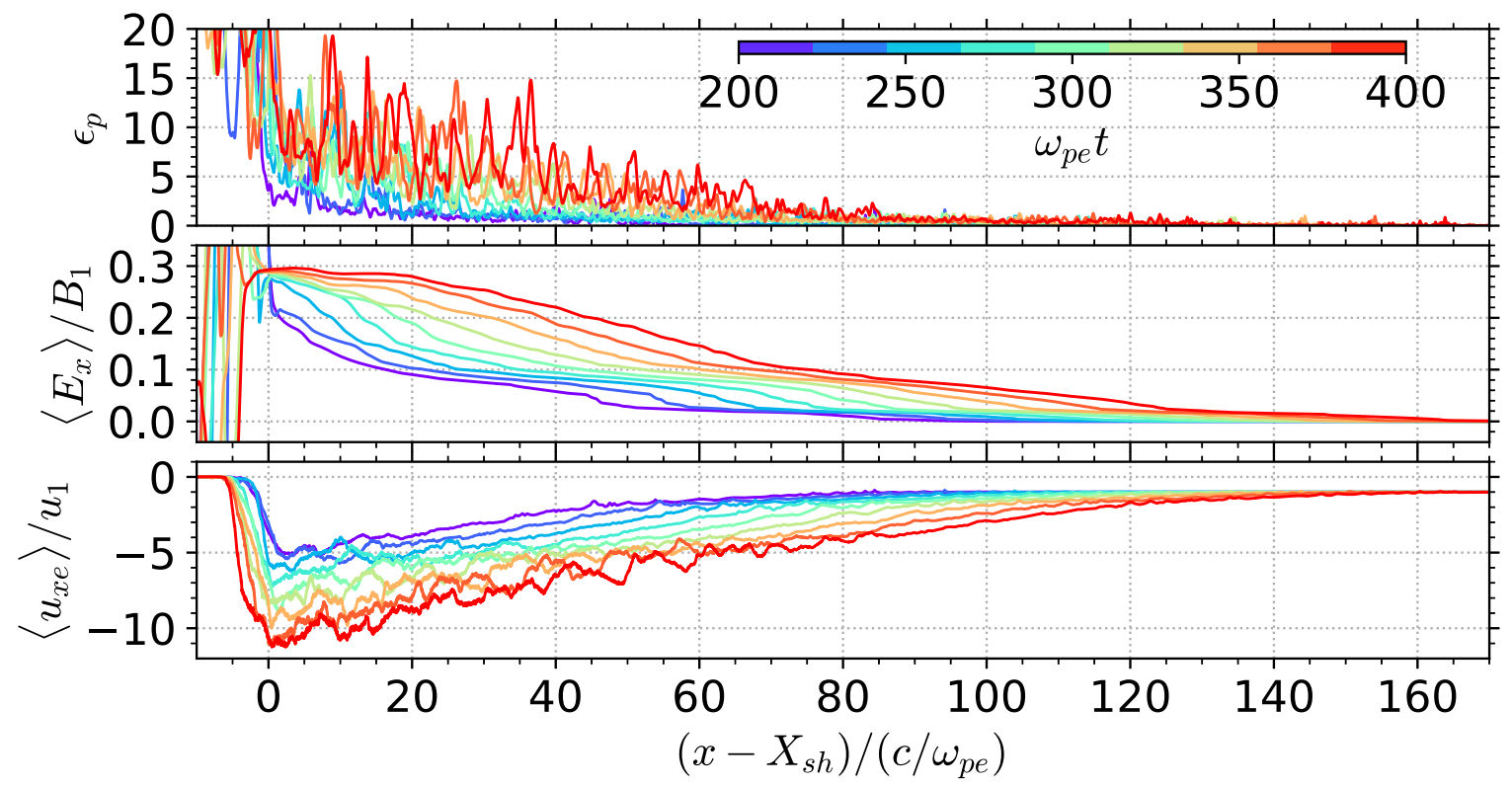

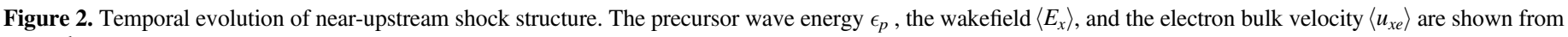
top to bottom.

be seen in the right panel of Figure $3, \epsilon_{p}$ is clearly proportional to $m_{i} / m_{e}$ for all $\sigma_{i}$. This result also supports our model. Equation (1) indicates that the total magnetization $\sigma_{i}$ dependence of the wave energy normalized by the total kinetic energy $\delta B^{2} / 4 \pi \gamma_{1} N_{1} m_{i} c^{2}$ shows the same tendency as that in pair plasmas. This is natural because the total kinetic energy is available for the SMI due to the ion-electron coupling. The precursor wave emission is well-characterized by the incoming total kinetic energy as long as the positive feedback process works.

In the case of the 2D shock at $\sigma_{e}=0.1$, we confirmed that the wakefield is excited by the large-amplitude precursor waves and that the wavelength of the laminar wakefield is consistent with the linear theory of the PDI as with the case of relatively high magnetization. However, the precursor wave amplitude is almost constant in the laminar-wakefield region and the positive feedback does not work. One of the reason for this discrepancy may be due to the inhomogeneity. The ion-scale fluctuations along the shock surface are generated at $\sigma_{e}=0.1$ (Sironi et al. 2013) and the inhomogeneity at the shock front is more prominent than that for $\sigma_{e}>1$. Consequently, the wave emission via the SMI may become less efficient at $\sigma_{e}=0.1$ (Iwamoto et al. 2017, 2018). For $\sigma_{e}>1$, the PDI rapidly grows and induces the positive electric field in the immediate upstream at an early stage of the shocks. Electrons enter the shock front during the acceleration phase and the positive feedback process then begins. On the other hand, for $\sigma_{e}=0.1$, the precursor wave slowly decays into the wakefield compared to that for $\sigma_{e}>0.1$ because the linear growth rate of the PDI is proportional to the amplitude of the pump wave (Kruer 1988). The wakefield is excited away from the shock front and the sinusoidal electric field is generated even in an early phase. The net acceleration of electrons is zero in the sinusoidal electric field and thus the positive feedback does not work at $\sigma_{e}=0.1$. If the positive feedback process does not work, the wave emission in ion-electron plasmas should be less efficient than that in pair plasmas because only electrons emit the precursor waves via the SMI.
Although the wave emission efficiency deteriorates at $\sigma_{e}=0.1$, this may not be necessarily the case for a sufficiently large mass ratio. We fixed the ion-to-electron mass ratio $m_{i} / m_{e}=50$ throughout the 2D simulations. Since the ratio of the ion gyroradius to the electron is equivalent to the mass ratio, the electron gyromotion is nearly unperturbed in the ionscale fluctuations for a sufficiently large mass ratio and the wave emission via the SMI would remain efficient at $\sigma_{e}=0.1$. If such a large mass ratio is used, ion Weibel instability would dominate the shock front even for high $\sigma_{e}$ and generate the ionscale magnetic field (Sironi et al. 2013). Electrons feel the Weibel-generated magnetic field and may emit the stronger precursor waves (Iwamoto et al. 2018). Therefore, we anticipate that the positive feedback process enhances the precursor wave emission for a wide range of $\sigma_{e}$ when the realistic mass ratio is used.

Since the incoming electrons are already thermalized before entering into the shock, the wave amplitude may decrease in time or might be completely shut off due to the suppression of the higher order harmonic excitation (Amato \& Arons 2006). The weak precursor waves would not excite the wakefields and the particle acceleration/heating may cease in a later phase. After the quiescent stage of the precursor waves, however, cold undisturbed electrons will enter the shock once again and the whole positive feedback cycle will be initiated. We thus speculate that this system may exhibit a cyclic behavior, which may periodically induce the large-amplitude electromagnetic waves.

\section{Particle Acceleration}

Figure 4 shows the energy spectra of electrons (blue) and ions (red) in the turbulent-wakefield region, where the wakefield collapses and the efficient particle acceleration occurs. The solid and dashed lines indicate the spectra for $\sigma_{e}=5$ and $\sigma_{e}=0.1$, respectively. The prime indicates physical quantities measured in the plasma rest frame. Note that we use the proper frame for comparison with the previous simulation results and laser experiments (Kuramitsu et al. 

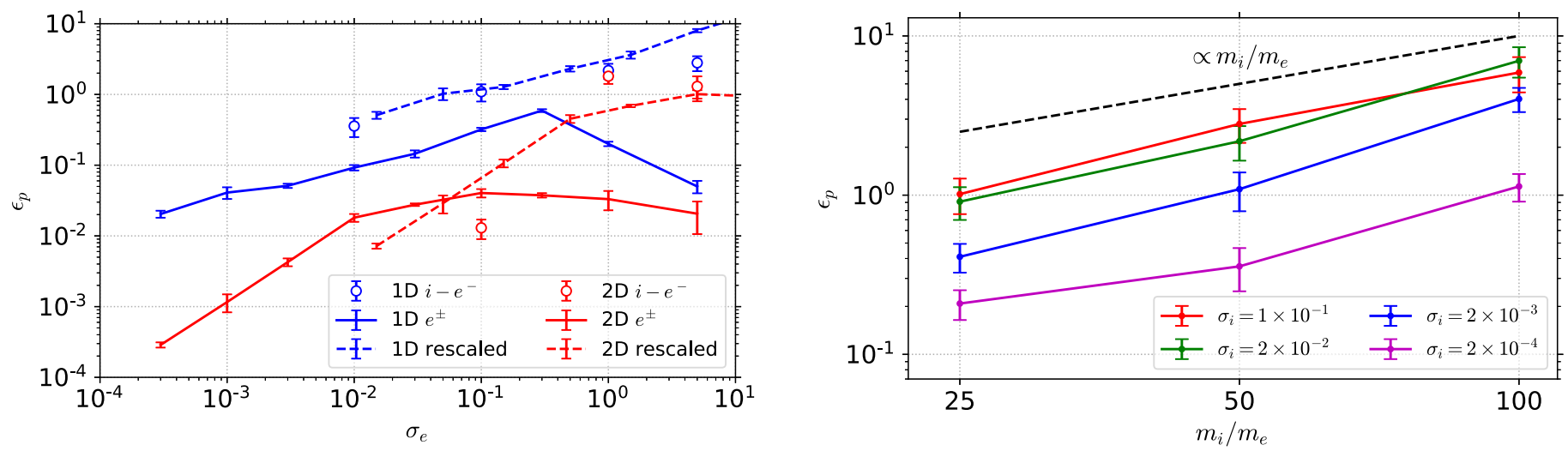

Figure 3. Left panel: the precursor wave energy $\epsilon_{p}$ in 1D (blue) and 2D (red) as a function of $\sigma_{e}$. The circles and solid lines indicate ion-electron and pair shock simulation results, respectively. The rescaled wave energy in pair plasmas is shown in the dashed lines. Right panel: the mass ratio dependence of the $1 \mathrm{D}$ wave energy $\epsilon_{p}$ at $\sigma_{i}=1 \times 10^{-1}$ (red), $2 \times 10^{-2}$ (green), $2 \times 10^{-3}$ (blue), and $2 \times 10^{-4}$ (magenta).

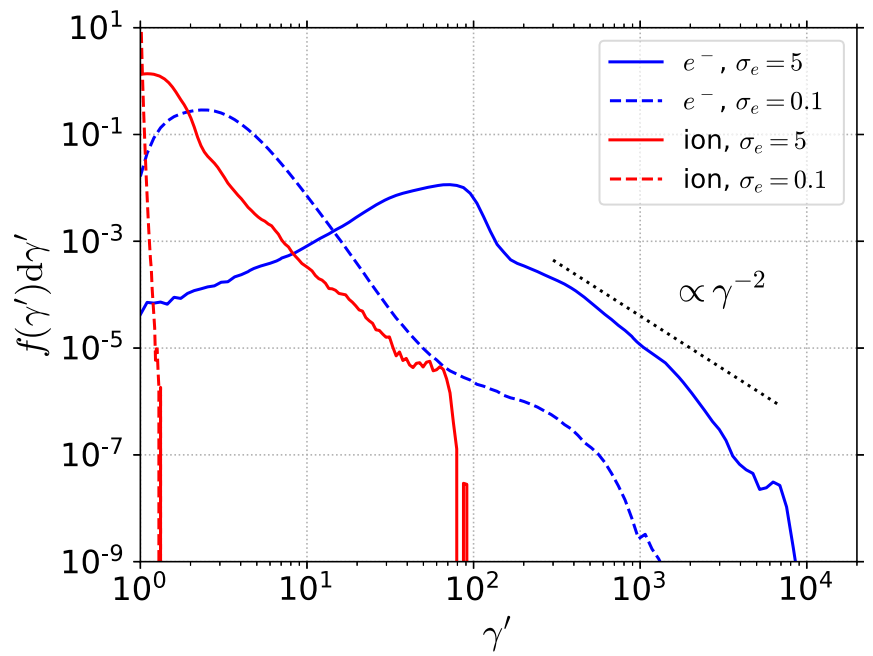

Figure 4. Near-upstream energy spectra of electrons (blue) and ions (red) for $\sigma_{e}=5$ (solid lines) and $\sigma_{e}=0.1$ (dashed lines) measured in the proper frame.

2008, 2011a, 2011b, 2012; Liu et al. 2017, 2018, 2019) where the spectra were measured in the plasma rest frame. We determined the bulk velocity in the turbulent-wakefield region and then the spectra are obtained by performing Lorentz transformation into the plasma rest frame. A power-law distribution $\propto \gamma^{-2}$ is also shown in black for reference. A clear nonthermal tail is observed for electrons in the case of $\sigma_{e}=5$, and the spectral index is close to 2. Surprisingly, nonthermal ions whose spectral index is close to 2 are also generated, which is a clear difference from the earlier studies. At $\sigma_{e}=0.1$, the wakefield amplitude is an order of magnitude smaller than $\sigma_{e}=5$ and thus ions are almost unaffected by such small wakefield. Electrons are accelerated, although less efficiently compared to $\sigma_{e}=5$, and the spectrum has the nonthermal component. The detailed acceleration mechanism will be presented in a future publication.

As already discussed in Section 4, we expect the cyclic excitation of the intense precursor waves in relativistic shocks. We thus think that the particle acceleration is not transient and that nonthermal particles may be periodically produced as well.

\section{Summary}

In this work, we have found that the precursor wave emission efficiency is dramatically enhanced for high $\sigma_{e}$ due to the positive feedback process associated with the ion-electron coupling. For low $\sigma_{e}$, the wave emission may be influenced by the ion-scale fluctuations unless a sufficiently large mass ratio is applied. The large-amplitude precursor wave initiates the nonlinear process of the PDI and the wakefield is destroyed in the near-upstream region. Nonthermal electrons and ions are generated inside the turbulent wakefield and the energy spectrum exhibits a power-law distribution. This study shows that the intense coherent wave emission and the particle acceleration can operate in relativistic astrophysical objects.

The authors would like to thank Shuichi Matsukiyo and Artem Bohdan for fruitful discussions.

This work used the computational resources of the HPCI system provided by Information Technology Center, Nagoya University through the HPCI System Research Project (Project ID: hp180071,hp180180).

This work is supported by Collaborative Research Project on Computer Science with High-Performance Computing in Nagoya University.

Numerical computations were in part carried out on Cray XC50 at Center for Computational Astrophysics, National Astronomical Observatory of Japan.

This work was supported in part by JSPS KAKENHI grant No. $17 \mathrm{H} 02877$.

The work of J.N., A.L., and O.K. has been supported by Narodowe Centrum Nauki through research project DEC2013/10/E/ST9/00662.

\section{ORCID iDs}

Masanori Iwamoto (ㄴ) https://orcid.org/0000-0003-2255-5229

Masahiro Hoshino (i) https://orcid.org/0000-0002-1818-9927

Jacek Niemiec $\odot$ https://orcid.org/0000-0001-6036-8569

Oleh Kobzar (1) https://orcid.org/0000-0001-6956-5884

Martin Pohl (1) https://orcid.org/0000-0001-7861-1707

\section{References}

Aab, A., Abreu, P., Aglietta, M., et al. 2015, ApJ, 804, 15 Aab, A., Abreu, P., Aglietta, M., et al. 2017, Sci, 357, 1266 Aab, A., Abreu, P., Aglietta, M., et al. 2018, ApJL, 853, L29 Aartsen, M. G., Ackermann, M., Adams, J., et al. 2018, Sci, 361, 147 Abbasi, R. U., Abe, M., Abu-Zayyad, T., et al. 2014, ApJL, 790, L21 Amato, E., \& Arons, J. 2006, ApJ, 653, 325

Arons, J. 2003, ApJ, 589, 871

Biermann, P. L., \& Strittmatter, P. A. 1987, ApJ, 322, 643 
Chen, P., Tajima, T., \& Takahashi, Y. 2002, PhRvL, 89, 161101

Fried, B. D. 1959, PhFl, 2, 337

Gallant, Y. A., Hoshino, M., Langdon, A. B., Arons, J., \& Max, C. E. 1992, ApJ, 391, 73

Hillas, A. M. 1984, ARA\&A, 22, 425

Hoshino, M. 2008, ApJ, 672, 940

Hoshino, M., \& Arons, J. 1991, PhFlB, 3, 818

Hoshino, M., Arons, J., Gallant, Y. A., \& Langdon, A. B. 1992, ApJ, 390, 454

Ikeya, N., \& Matsumoto, Y. 2015, PASJ, 67, 64

Iwamoto, M., Amano, T., Hoshino, M., \& Matsumoto, Y. 2017, ApJ, 840, 52

Iwamoto, M., Amano, T., Hoshino, M., \& Matsumoto, Y. 2018, ApJ, 858, 93

Kruer, W. L. 1988, The Physics of Laser Plasma Interactions (Boston: Addison-Wesley)

Kuramitsu, Y., Nakanii, N., Kondo, K., et al. 2011a, PhPl, 18, 010701

Kuramitsu, Y., Nakanii, N., Kondo, K., et al. 2011b, PhRvE, 83, 026401

Kuramitsu, Y., Sakawa, Y., Hoshino, M., Chen, S. H., \& Takabe, H. 2012 HEDP, 8, 266

Kuramitsu, Y., Sakawa, Y., Kato, T., Takabe, H., \& Hoshino, M. 2008, ApJ, 682, 113

Langdon, A. B., Arons, J., \& Max, C. E. 1988, PhRvL, 61, 779

Liu, Y. L., Isayama, S., Chen, S. H., \& Kuramitsu, Y. 2019, HEDP, 31, 64

Liu, Y. L., Kuramitsu, Y., Isayama, S., \& Chen, S. H. 2018, PhPl, 25, 013110
Liu, Y. L., Kuramitsu, Y., Moritaka, T., \& Chen, S. H. 2017, HEDP, 22, 46 Lyubarsky, Y. 2006, ApJ, 652, 1297

Lyubarsky, Y. 2014, MNRAS, 442, L9

Lyubarsky, Y. 2018, MNRAS, 474, 1135

Marscher, A. P. 2006, in AIP Conf. Proc. 856, RELATIVISTIC JETS: The Common Physics of AGN, Microquasars, and Gamma-Ray Bursts, ed. P. A. Hughes \& J. N. Bregman (Ann Arbor, Michigan: AIP), 1

Matsumoto, Y., Amano, T., \& Hoshino, M. 2013, PhRvL, 111, 215003

Matsumoto, Y., Amano, T., Kato, T. N., \& Hoshino, M. 2015, Sci, 347, 974

Metzger, B. D., Margalit, B., \& Sironi, L. 2019, MNRAS, 485, 4091

Milgrom, M., \& Usov, V. 1995, ApJL, 449, L37

Mima, K., \& Nishikawa, K. 1984, in Basic Plasma Physics, ed. A. A. Galeev \& R. N. Sudan (Amsterdam: North-Holland)

Murase, K., Mészáros, P., \& Zhang, B. 2009, PhRvD, 79, 103001

Piran, T. 2005, RvMP, 76, 1143

Plotnikov, I., Grassi, A., \& Grech, M. 2018, MNRAS, 477, 5238

Plotnikov, I., \& Sironi, L. 2019, MNRAS, 485, 3816

Sironi, L., Spitkovsky, A., \& Arons, J. 2013, ApJ, 771, 54

Tajima, T., \& Dawson, J. M. 1979, PhRvL, 43, 267

Vietri, M. 1995, ApJ, 453, 883

Waxman, E. 1995, ApJL, 452, L1

Weibel, E. S. 1959, PhRvL, 2, 83 